\title{
Hyers-Ulam stability of nonlinear impulsive Volterra integro-delay dynamic system on time scales
}

\author{
Akbar Zada ${ }^{\mathrm{a}}$, Syed Omar Shah" ${ }^{\mathrm{a}}$, Yongjin Lib,* \\ ${ }^{a}$ Department of Mathematics, University of Peshawar, Peshawar 25000, Pakistan. \\ ${ }^{b}$ Department of Mathematics, Sun Yat-sen University, Guangzhou, 510275, P. R. China. \\ Communicated by S.-M. Jung
}

\begin{abstract}
This paper proves the Hyers-Ulam stability and Hyers-Ulam-Rassias stability of nonlinear impulsive Volterra integro-delay dynamic system on time scales via a fixed point approach. The uniqueness and existence of the solution of nonlinear impulsive Volterra integro-delay dynamic system is proved with the help of Picard operator. The main tools for proving our results are abstract Grönwall lemma and Banach contraction principle. We also make some assumptions along with Lipschitz condition which make our results appropriate for the approach we are using. (C)2017 All rights reserved.
\end{abstract}

Keywords: Hyers-Ulam stability, Hyers-Ulam-Rassias stability, time scale, nonlinear Volterra integro-delay dynamic system. 2010 MSC: $34 \mathrm{~N} 05,34 \mathrm{~A} 37,45 \mathrm{M} 10,45 \mathrm{~J} 05$.

\section{Introduction}

Let $(\mathrm{G}, *)$ and $(\mathrm{H},$.$) be groups, then a function \phi:(\mathrm{G}, *) \rightarrow(\mathrm{H},$.$) is said to be a group homomorphism$ if it is given by

$$
\phi(x * y)=\phi(x) \cdot \phi(y), \quad \forall x, y \in G .
$$

Ulam [23, 24] considered $(\mathrm{H}, . . \mathbf{d})$ a metric group with metric $\mathbf{d}(.,$.$) and inquired a question, if for any$ $\epsilon>0$ and $\phi:(\mathrm{G}, *) \rightarrow(\mathrm{H},$.$) satisfies the inequality$

$$
\mathbf{d}(\phi(x * y), \phi(x) . \phi(y)) \leqslant \epsilon, \quad \forall x, y \in G,
$$

then for an approximate homomorphism $\psi:(\mathrm{G}, *) \rightarrow(\mathrm{H},$.$) can we find a real number \delta>0$ such that

$$
\mathbf{d}(\phi(x), \psi(x)) \leqslant \delta, \quad \forall x \in \mathrm{G} .
$$

To deal this problem, Hyers [10] using direct method, brilliantly gave a partial answer to the case of functional equation by considering $\mathrm{G}$ and $\mathrm{H}$ to be Banach spaces. Afterward, it was called the Hyers-Ulam

\footnotetext{
${ }^{*}$ Corresponding author

Email addresses: zadababo@yahoo.com, akbarzada@uop.edu.pk (Akbar Zada), omarshah89@yahoo.com, omarshahstd@uop.edu.pk (Syed Omar Shah), stslyj@mail.sysu.edu.cn (Yongjin Li)

doi:10.22436/jnsa.010.11.08
} 
problem and the study of this area has grown-up to be one of the important subjects in mathematical analysis. In 1978, Rassias [21] provided an extension of the Hyers-Ulam stability by introducing new function variables. As a result, another new stability concept, Hyers-Ulam-Rassias stability, was named by mathematicians.

In the literature, many researchers paid attention to the stability properties of different kinds of differential equations. We emphasize that Ulam's type stability problems have been taken up by a huge amount of mathematicians and the study of this region has grown-up to be one of the vital subjects in mathematical analysis. However, among the functional equations, Obłoza seems to be the first mathematician who has investigated the Hyers-Ulam stability of linear differential equations (see [18, 19]). Thereafter, Alsina and Ger published their paper which handles the Hyers-Ulam stability of the linear differential equation $y^{\prime}(t)=y(t)$. They proved that if a differentiable function $y(t)$ is a solution of the inequality $\left|y^{\prime}(t)-y(t)\right| \leqslant \varepsilon$ for some $\varepsilon>0$ and for all $t \in(a, \infty)$, then there exists a constant $c$ such that $\left|y(t)-c e^{t}\right| \leqslant 3 \varepsilon$ for all $t \in(a, \infty)$, where $a \in \mathbb{R}$ ([2]). Jung in 2004 [11] investigated Hyers-Ulam stability of first order linear differential equations. In 2010, Li and Shen [15] studied the Hyers-Ulam stability of linear differential equations of second order. For more details on Hyers-Ulam stability, see [12-14, 16, 18, 25, 26, 28-31].

Many real world phenomena are represented by smooth differential equations. However, the situation becomes quite different in the case when a physical phenomena has sudden changes in its state such as mechanical systems with impact, biological systems with heart beats, blood flows, population dynamics, theoretical physics and so on (see [4]). Adequate mathematical models of such processes are systems of differential equations with impulses i.e., impulsive differential equations. An impulsive differential equation is described by three components: a continuous time differential equation, which governs the state of the system between impulses; an impulse equation, which models an impulsive jump defined by a jump function at the instant an impulse occurs; and a jump criterion, which defines a set of jump events in which the impulse equation is active.

The theory of dynamic equations on time scale has been developing rapidly and has received a lot of attention in recent years. This theory was introduced by Hilger [9] in 1990, with the motivation of providing a unification to continuous and discrete calculus. For more details on time scale, see [58, 17, 20, 27]. In 2013, András and Mészáros [3] obtained some results about the Hyers-Ulam stability of some integral equations on time scale via Picard operators. Agarwal et al. [1] in 2014, discussed some results about the stability of linear impulsive Volterra integro-dynamic system on time scales. To the best of our knowledge, only few papers are devoted to the stability of impulsive Volterra integro-dynamic systems. However, as far as we know, the Hyers-Ulam stability and Hyers-Ulam-Rassias stability of nonlinear impulsive Volterra integro-delay dynamic systems have not been studied yet.

In this paper, we obtain Hyers-Ulam stability and Hyers-Ulam-Rassias stability of nonlinear impulsive Volterra integro-delay dynamic system of the form

$$
\left\{\begin{array}{l}
z^{\Delta}(\mathrm{t})=M(\mathrm{t}) z(\mathrm{t})+\int_{\mathrm{t}_{0}}^{\mathrm{t}} \mathcal{K}(\mathrm{t}, \mathrm{s}, z(\mathrm{~s}), z(\mathrm{~h}(\mathrm{~s}))) \Delta s, \quad \mathrm{t} \in \mathcal{T}_{\mathcal{S}}{ }^{\prime}=\mathcal{T}_{\mathcal{S}}{ }^{0} \backslash\left\{\mathrm{t}_{1}, \mathrm{t}_{2}, \cdots, \mathrm{t}_{\mathrm{m}}\right\}, \\
\Delta z\left(\mathrm{t}_{\mathrm{k}}\right)=z\left(\mathrm{t}_{\mathrm{k}}^{+}\right)-z\left(\mathrm{t}_{\mathrm{k}}^{-}\right)=\Upsilon_{\mathrm{k}}\left(z\left(\mathrm{t}_{\mathrm{k}}^{-}\right)\right), \quad \mathrm{k}=1,2, \cdots, \mathrm{m}, \\
z(\mathrm{t})=\alpha(\mathrm{t}), \quad \mathrm{t} \in\left[\mathrm{t}_{0}-\lambda, \mathrm{t}_{0}\right], \\
z\left(\mathrm{t}_{0}\right)=\alpha\left(\mathrm{t}_{0}\right)=z_{0},
\end{array}\right.
$$

where $\lambda>0, M(t)$ is piecewise continuous and a regressive square matrix of order $m$ on $\mathcal{T}_{\mathcal{S}}{ }^{0}:=\left[t_{0}, t_{f}\right]_{\mathcal{T}_{\mathcal{S}}}^{z}$ $t_{f}>t_{0} \geqslant 0$ and $\mathcal{K}(t, s, z(s), z(h(s)))$ is piecewise continuous operator on

$$
\Gamma=\left\{(\mathrm{t}, \mathrm{s}, z): \mathrm{t}_{0} \leqslant \mathrm{~s} \leqslant \mathrm{t} \leqslant \mathrm{t}_{\mathrm{f}}, z \in \mathbb{R}^{\mathrm{m}}\right\} .
$$

Also $\Upsilon_{k}: \mathbb{R} \rightarrow \mathbb{R}, \alpha:\left[t_{0}-\lambda, t_{0}\right] \rightarrow \mathbb{R}$ are continuous functions, $z\left(t_{k}^{+}\right)=\lim _{\tau \rightarrow 0^{+}} z\left(t_{k}+\tau\right)$ and $z\left(t_{k}^{-}\right)=$ $\lim _{\tau \rightarrow 0^{+}} z\left(t_{k}-\tau\right)$ are respectively the right and left side limits of $z(t)$ at $t_{k}$, where $t_{k}$ satisfies

$$
\mathrm{t}_{0}<\mathrm{t}_{1}<\mathrm{t}_{3}<\cdots<\mathrm{t}_{\mathrm{m}}<\mathrm{t}_{\mathrm{m}+1}=\mathrm{t}_{\mathrm{f}}<+\infty .
$$


Moreover, $h: \mathcal{T}_{\mathcal{S}}{ }^{0} \rightarrow \mathcal{T}_{\mathcal{S}}{ }^{0} \cap\left[t_{0}-\lambda, t_{0}\right]$ is a continuous delay function such that $h(t) \leqslant t$.

\section{Preliminaries}

The time scale is defined to be any nonempty closed subset of real numbers and is denoted by $\mathcal{T}_{\mathcal{S}}$. The forward jump operator $\Theta: \mathcal{T}_{\mathcal{S}} \rightarrow \mathcal{T}_{\mathcal{S}}$, backward jump operator $\rho: \mathcal{T}_{\mathcal{S}} \rightarrow \mathcal{T}_{\mathcal{S}}$ and graininess function $\mu: \mathcal{T}_{\mathcal{S}} \rightarrow[0, \infty)$ are respectively defined as:

$$
\Theta(s)=\inf \left\{t \in \mathcal{T}_{\mathcal{S}}: t>s\right\}, \quad \rho(s)=\sup \left\{t \in \mathcal{T}_{\mathcal{S}}: t<s\right\}, \quad \mu(s)=\Theta(s)-s .
$$

For any $t \in \mathcal{T}_{\mathcal{S}}$, if $t<\rho(t)$ then point $t$ is said to be left-scattered and if $t=\rho(t)$ then $t$ is called left-dense. If $t<\Theta(t)$ and $\Theta(t)=t$, then point $t \in \mathcal{T}_{\mathcal{S}}$ is called right-scattered and right-dense, respectively. The set $\mathcal{T}_{\mathcal{S}}{ }^{z}$ is known as derived form of time scale $\mathcal{T}_{\mathcal{S}}$ and is defined as:

$$
\mathcal{T}_{\mathcal{S}}{ }^{z}= \begin{cases}\mathcal{T}_{\mathcal{S}} \backslash\left(\rho\left(\sup \mathcal{T}_{\mathcal{S}}\right), \sup \mathcal{T}_{\mathcal{S}}\right], & \text { if } \sup \mathcal{T}_{\mathcal{S}}<\infty, \\ \mathcal{T}_{\mathcal{S}}, & \text { if } \sup \mathcal{T}_{\mathcal{S}}=\infty .\end{cases}
$$

The real-valued function $W: \mathcal{T}_{\mathcal{S}} \rightarrow \mathbb{R}$ is called right-dense continuous, if it is continuous at every rightdense point on $\mathcal{T}_{\mathcal{S}}$ and its left-sided limit exists at every left-dense point on $\mathcal{T}_{\mathcal{S}}$. The real-valued function $W: \mathcal{T}_{\mathcal{S}} \rightarrow \mathbb{R}$ is called regressive, if $1+\mu(t) W(t) \neq 0$, for all $t \in \mathcal{T}_{\mathcal{S}}{ }^{z}$ and if $1+\mu(t) W(t)>0$, then $W$ is called positively regressive. The set of all right-dense continuous and regressive, right-dense continuous and positively regressive functions, respectively, will be denoted by $\mathcal{R}_{\mathcal{G}}\left(\mathcal{T}_{\mathcal{S}}\right)$ and $\mathcal{R}_{\mathcal{G}}\left(\mathcal{T}_{\mathcal{S}}\right)^{+}$. The delta derivative of the function $W: \mathcal{T}_{\mathcal{S}} \rightarrow \mathbb{R}$ at $t \in \mathcal{T}_{\mathcal{S}}{ }^{z}$ is defined by

$$
W^{\Delta}(t)=\lim _{s \rightarrow t, s \neq \Theta(t)} \frac{W(\Theta(t))-W(s)}{\Theta(t)-s} .
$$

The $\Delta$-integral of the rd-continuous function $W: \mathcal{T}_{\mathcal{S}} \rightarrow \mathbb{R}$ is defined by

$$
\int_{\mathrm{a}}^{\mathrm{b}} \mathrm{W}(\mathrm{t}) \Delta \mathrm{t}=w(\mathrm{~b})-w(\mathrm{a}), \quad \forall \mathrm{a}, \mathrm{b} \in \mathcal{T}_{\mathcal{S}},
$$

where the rd-continuous function $w$ is an anti-derivative of $W$, i.e., $w^{\Delta}=W$ on $\mathcal{T}_{\mathcal{S}}{ }^{z}$.

The generalized exponential function $e_{W}(a, b)$ for $W \in \mathcal{R}_{\mathcal{G}}\left(\mathcal{T}_{\mathcal{S}}\right)$ on $\mathcal{T}_{\mathcal{S}}$ is defined as

$$
e_{W}(a, b)=\exp \left(\int_{a}^{b} \Phi_{\mu(s)} W(s) \Delta s\right), \quad \forall a, b \in \mathcal{T}_{\mathcal{S}},
$$

where

$$
\Phi_{\mu(t)} W(t)= \begin{cases}\frac{\log (1+\mu(t) W(t))}{\mu(t)}, & \text { if } \mu(t) \neq 0, \\ W(t), & \text { if } \mu(t)=0,\end{cases}
$$

is the cylindrical transformation.

The fundamental matrix is defined to be the general solution to the matrix dynamic equation $z^{\Delta}(t)=$ $M(t) z(t), z\left(t_{0}\right)=z_{0}, t \in \mathcal{T}_{\mathcal{S}}^{0}$ and is denoted by $\Psi_{M}\left(t, t_{0}\right)$.

Consider the metric space $\mathcal{T}_{\mathcal{S} 1} \times \mathcal{T}_{\mathcal{S} 2}=\left\{(\mathrm{m}, \mathrm{n}): \mathrm{m} \in \mathcal{T}_{\mathcal{S} 1}, \mathrm{n} \in \mathcal{T}_{\mathcal{S}_{2}}\right\}$ which is a complete metric space with the metric defined by

$$
\mathbf{d}\left(\left(m_{1}, n_{1}\right),\left(m_{2}, n_{2}\right)\right)=\sqrt{\left(m_{1}-m_{2}\right)^{2}+\left(n_{1}-n_{2}\right)^{2}}, \quad\left(m_{1}, n_{1}\right),\left(m_{2}, n_{2}\right) \in \mathcal{T}_{\mathcal{S} 1} \times \mathcal{T}_{\mathcal{S} 2},
$$

where $\mathcal{T}_{\mathcal{S}_{1}}$ and $\mathcal{T}_{\mathcal{S}_{2}}$ are the time scales. 
The function $W: \mathcal{T}_{\mathcal{S}_{1}} \times \mathcal{T}_{\mathcal{S}_{2}} \rightarrow \mathbb{R}$ is said to be continuous at $(m, n) \in \mathcal{T}_{\mathcal{S}_{1}} \times \mathcal{T}_{\mathcal{S}_{2}}$ if for every $\epsilon>0$ there exists $\delta>0$ such that $\left\|g(m, n)-g\left(m_{0}, n_{0}\right)\right\|<\epsilon$ for all $\left(m_{0}, n_{0}\right) \in \mathcal{T}_{\mathcal{S}_{1}} \times \mathcal{T}_{\mathcal{S}_{2}}$ satisfying

$$
\mathbf{d}\left((\mathrm{m}, \mathrm{n}),\left(\mathrm{m}_{0}, \mathrm{n}_{0}\right)\right)<\delta .
$$

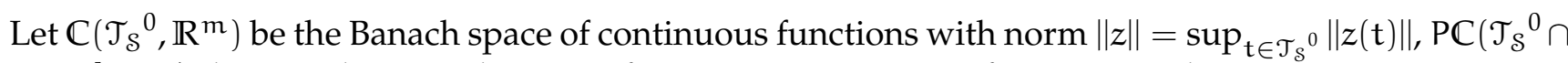
$\left.\left[t_{0}-\lambda, t_{0}\right], \mathbb{R}^{m}\right)$ denotes the Banach space of piecewise continuous functions with norm

$$
\|z\|=\sup _{t \in \mathcal{T}_{\mathcal{S}}^{0} \cap\left[\mathrm{t}_{0}-\lambda, \mathrm{t}_{0}\right]}\|z(\mathrm{t})\|,
$$

and $\mathrm{PC}^{1}\left(\mathcal{T}_{\mathcal{S}}{ }^{0}, \mathbb{R}^{\mathrm{m}}\right)=\left\{z \in \mathrm{PC}\left(\mathcal{T}_{\mathcal{S}}^{0} \cap\left[\mathrm{t}_{0}-\lambda, \mathrm{t}_{0}\right], \mathbb{R}^{\mathrm{m}}\right): z^{\Delta} \in \mathrm{PC}\left(\mathcal{T}_{\mathcal{S}}{ }^{0} \cap\left[\mathrm{t}_{0}-\lambda, \mathrm{t}_{0}\right], \mathbb{R}^{\mathrm{m}}\right)\right\}$ is Banach space with norm $\|z\|_{\mathrm{PC}^{1}}=\max \left\{\|z\|_{\mathrm{PC}},\left\|z^{\Delta}\right\|_{\mathrm{PC}}\right\}$. Consider the following inequalities,

$$
\begin{aligned}
& \left\{\begin{array}{l}
\left\|y^{\Delta}(t)-M(t) y(t)-\int_{t_{0}}^{t} \mathcal{K}(t, s, y(s), y(h(s))) \Delta s\right\| \leqslant \epsilon, \quad t \in \mathcal{T}_{\mathcal{S}}{ }^{\prime}, \\
\left\|\Delta y\left(t_{k}\right)-\Upsilon_{k}\left(y\left(t_{k}^{-}\right)\right)\right\| \leqslant \epsilon, \quad k=1,2, \cdots, m,
\end{array}\right. \\
& \left\{\begin{array}{l}
\left\|y^{\Delta}(t)-M(t) y(t)-\int_{t_{0}}^{t} \mathcal{K}(t, s, y(s), y(h(s))) \Delta s\right\| \leqslant \varphi(t), \quad t \in \mathcal{T}_{\mathcal{S}}{ }^{\prime}, \\
\left\|\Delta y\left(t_{k}\right)-\Upsilon_{k}\left(y\left(t_{k}^{-}\right)\right)\right\| \leqslant k, \quad k=1,2, \cdots, m,
\end{array}\right.
\end{aligned}
$$

where $\varphi \in \mathbb{C}\left(\mathcal{T}_{\mathcal{S}}{ }^{0}, \mathbb{R}^{m}\right)$ is an increasing function.

Definition 2.1. Equation (1.1) is Hyers-Ulam stable on $\mathcal{T}_{\mathcal{S}}{ }^{0} \cap\left[\mathrm{t}_{0}-\lambda, \mathrm{t}_{0}\right]$ if for every

$$
y \in \operatorname{PC}\left(\mathcal{T}_{\mathcal{S}}^{0} \cap\left[\mathrm{t}_{0}-\lambda, \mathrm{t}_{0}\right], \mathbb{R}^{\mathrm{m}}\right) \cap \mathrm{PC}^{1}\left(\mathcal{T}_{\mathcal{S}}{ }^{0}, \mathbb{R}^{\mathrm{m}}\right),
$$

satisfying (2.1), there exists a solution $y_{0} \in \operatorname{PC}\left(\mathcal{T}_{\mathcal{S}}^{0} \cap\left[t_{0}-\lambda, t_{0}\right], \mathbb{R}^{m}\right) \cap P C^{1}\left(\mathcal{T}_{\mathcal{S}}^{0}, \mathbb{R}^{m}\right)$ of $(1.1)$ with

$$
\left\|y_{0}(t)-y(t)\right\| \leqslant K \epsilon, \quad K>0, \quad \forall t \in \mathcal{T}_{\mathcal{S}}^{0} \cap\left[t_{0}-\lambda, t_{0}\right] .
$$

Definition 2.2. Equation (1.1) is Hyers-Ulam-Rassias stable on $\mathcal{T}_{\mathcal{S}}{ }^{0} \cap\left[\mathrm{t}_{0}-\lambda, \mathrm{t}_{0}\right]$ if for every

$$
y \in \operatorname{PC}\left(\mathcal{T}_{\mathcal{S}}^{0} \cap\left[t_{0}-\lambda, t_{0}\right], \mathbb{R}^{m}\right) \cap \operatorname{PC}^{1}\left(\mathcal{T}_{\mathcal{S}}^{0}, \mathbb{R}^{m}\right),
$$

satisfying (2.2), there exists a solution $y_{0} \in \operatorname{PC}\left(\mathcal{T}_{\mathcal{S}}^{0} \cap\left[t_{0}-\lambda, t_{0}\right], \mathbb{R}^{m}\right) \cap P C^{1}\left(\mathcal{T}_{\mathcal{S}}^{0}, \mathbb{R}^{m}\right)$ of $(1.1)$ with

$$
\left\|y_{0}(t)-y(t)\right\| \leqslant K \varphi(t), \quad K>0, \quad \forall t \in \mathcal{T}_{\mathcal{S}}^{0} \cap\left[t_{0}-\lambda, t_{0}\right]
$$

Definition 2.3. Let $(X ; \mathbf{d})$ be any metric space. An operator $\Lambda: X \rightarrow X$ is a Picard operator, if it has a unique fixed point $\mathbf{x}^{*} \in \mathcal{X}$ such that for all $\mathbf{x} \in \mathcal{X},\left\{\Lambda^{(\mathrm{n})}(\mathrm{x})\right\} \rightarrow \mathbf{x}^{*}$ as $\mathrm{n} \rightarrow \infty$.

Lemma 2.4 ([17]). Let $\tau \in \mathcal{T}_{\mathcal{S}}^{+}, y, b \in \mathcal{R}_{\mathcal{G}}\left(\mathcal{T}_{\mathcal{S}}{ }^{+}\right), p \in \mathcal{R}_{\mathcal{G}}\left(\mathcal{T}_{\mathcal{S}}{ }^{+}\right)^{+}$and $c, b_{k} \in \mathbb{R}^{+}, k=1,2, \cdots$, then

$$
y(t) \leqslant c+\int_{\tau}^{t} p(s) y(s) \Delta s+\sum_{\tau<t_{k}<t} b_{k} y\left(t_{k}\right),
$$

implies

$$
y(t) \leqslant c \prod_{\tau<t_{k}<t}\left(1+b_{k}\right) e_{p}(t, \tau), \quad t \geqslant \tau
$$

Lemma 2.5 (Abstract Grönwall Lemma [22]). Let $(X, \mathbf{d}, \leqslant)$ be an ordered metric space and $\wedge: X \rightarrow X$ be an increasing Picard operator with fixed point $\mathbf{x}^{*}$. Then for any $\mathbf{x} \in X, \mathbf{x} \leqslant \Lambda(\mathbf{x})$ implies $\mathbf{x} \leqslant \mathbf{x}^{*}$ and $\mathbf{x} \geqslant \Lambda(\mathbf{x})$ implies $\mathbf{x} \geqslant \mathbf{x}^{*}$. 
Remark 2.6. A function

$$
y \in \operatorname{PC}^{1}\left(\mathcal{T}_{\mathcal{S}}^{0}, \mathbb{R}^{\mathrm{m}}\right),
$$

satisfies (2.1) if and only if there is a function $f \in P C\left(\mathcal{T}_{\mathcal{S}}^{0} \cap\left[t_{0}-\lambda, t_{0}\right], \mathbb{R}^{m}\right)$ and a sequence $f_{k}$ (which depends on y) such that $\|f(t)\| \leqslant \epsilon$ for all $t \in \mathcal{T}_{\mathcal{S}}^{0} \cap\left[t_{0}-\lambda, t_{0}\right],\left\|f_{k}\right\| \leqslant \epsilon$ for all $k=1,2, \cdots$, $m$, and

$$
\left\{\begin{array}{l}
y^{\Delta}(t)=M(t) y(t)+\int_{t_{0}}^{t} \mathcal{K}(t, s, y(s), y(h(s))) \Delta s+f(t), \quad y\left(t_{0}\right)=y_{0}, \quad t \in \mathcal{T}_{\mathcal{S}}^{\prime}, \\
\Delta y\left(t_{k}\right)=\Upsilon_{k}\left(y\left(t_{k}^{-}\right)\right)+f_{k}, \quad k=1,2, \cdots, m .
\end{array}\right.
$$

We have similar remark for (2.2).

Lemma 2.7. Every $y \in \mathrm{PC}^{1}\left(\mathcal{T}_{\mathcal{S}}{ }^{0}, \mathbb{R}^{\mathrm{m}}\right)$ that satisfies (1.1) also comes out perfect on the following inequality:

$$
\left\|y(t)-\Psi_{M}\left(t, t_{0}\right) y_{0}-\sum_{j=1}^{k} \Upsilon\left(y\left(t_{j}^{-}\right)\right)-\int_{t_{0}}^{t} \Psi_{M}(t, \Theta(s)) \int_{t_{0}}^{s} \mathcal{K}(s, u, y(u), y(h(u))) \Delta u \Delta s\right\| \leqslant\left(k+t_{f}-t_{0}\right) \epsilon
$$

for $\mathrm{t} \in\left(\mathrm{t}_{\mathrm{k}}, \mathrm{t}_{\mathrm{k}+1}\right] \subset \mathcal{T}_{\mathcal{S}}^{0}$.

Proof. If $y \in P C^{1}\left(\mathcal{T}_{\mathcal{S}}{ }^{0}, \mathbb{R}^{\mathrm{m}}\right)$ satisfies (2.1), then by Remark 2.6, we have

$$
\left\{\begin{array}{l}
y^{\Delta}(t)=M(t) z(t)+\int_{t_{0}}^{t} \mathcal{K}(t, s, z(s), z(h(s))) \Delta s+f(t), \quad t \in \mathcal{T}_{\mathcal{S}}^{\prime} \\
\Delta y\left(t_{k}\right)=\Upsilon_{k}\left(y\left(t_{k}^{-}\right)\right)+f_{k}, \quad k=1,2, \cdots, m
\end{array}\right.
$$

Then

$$
y(t)=y_{0}+\Psi_{M}\left(t, t_{0}\right) y_{0}+\sum_{j=1}^{k} \Upsilon\left(y\left(t_{j}^{-}\right)\right)+\sum_{i=1}^{k} f_{i}+\int_{t_{0}}^{t} \Psi_{M}(t, \Theta(s)) \int_{t_{0}}^{s} \mathcal{K}(s, u, y(u), y(h(u))) \Delta u \Delta s+\int_{t_{0}}^{t} f(s) \Delta s .
$$

So,

$$
\begin{aligned}
\| y(t) & -y_{0}-\Psi_{M}\left(t, t_{0}\right) y_{0}-\sum_{j=1}^{k} \Upsilon\left(y\left(t_{j}^{-}\right)\right)-\int_{t_{0}}^{t} \Psi_{M}(t, \Theta(s)) \int_{t_{0}}^{s} \mathcal{K}(s, u, y(u), y(h(u))) \Delta u \Delta s \| \\
& \leqslant \int_{t_{0}}^{t}\|f(s)\| \Delta s+\sum_{i=1}^{k}\left\|f_{i}\right\| \\
& \leqslant\left(k+t-t_{0}\right) \epsilon \\
& \leqslant\left(k+t_{f}-t_{0}\right) \epsilon .
\end{aligned}
$$

We have similar remarks for (2.2).

\section{Main results}

Now we are going to give our result on Hyers-Ulam stability.

Theorem 3.1. If

(a) The function $\mathcal{K}$ is piecewise continuous with the Lipschitz condition

$$
\left\|\mathcal{K}\left(t, s, x_{1}, x_{2}\right)-\mathcal{K}\left(t, s, y_{1}, y_{2}\right)\right\| \leqslant \sum_{i=1}^{2} L\left\|x_{i}-y_{i}\right\|, \quad L>0
$$

for $\mathrm{t}_{0} \leqslant \mathrm{~s} \leqslant \mathrm{t} \leqslant \mathrm{t}_{\mathrm{f}}$, and for all $\mathrm{x}_{\mathrm{i}}, \mathrm{y}_{\mathrm{i}} \in \mathbb{R}^{\mathrm{m}}, \mathrm{i} \in\{1,2\}$; 
(b) $\Upsilon_{k}: \mathbb{R} \rightarrow \mathbb{R}$ is such that $\left\|\Upsilon_{k}\left(x_{1}\right)-\Upsilon_{k}\left(x_{2}\right)\right\| \leqslant M_{k}\left\|x_{1}-x_{2}\right\|, M_{k}>0$, for all $k \in\{1,2, \cdots, m\}$ and $x_{1}, x_{2} \in \mathbb{R}, i \in\{1,2\}$;

(c) $\left(\sum_{j=1}^{m} M_{j}+2 \sup _{t \in \mathcal{T}_{\mathcal{S}}^{0} \cap\left[\mathrm{t}_{0}-\lambda, \mathrm{t}_{0}\right]} \int_{\mathrm{t}_{0}}^{\mathrm{t}}\left\|\Psi_{M}(\mathrm{t}, \Theta(s))\right\| \int_{\mathrm{t}_{0}}^{\mathrm{s}} \mathrm{L} \Delta \mathrm{u} \Delta \mathrm{s}\right)<1$;

(d) for some $\mathrm{C}_{\mathrm{k}} \geqslant 0$, we have $\left\|\Psi_{M}(\mathrm{t}, \Theta(\mathrm{s}))\right\|=\sup _{\mathrm{t} \in \mathcal{T}_{\mathcal{S}}^{0} \cap\left[\mathrm{t}_{0}-\lambda, \mathrm{t}_{0}\right]}\left\|\Psi_{M}(\mathrm{t}, \Theta(\mathrm{s}))\right\| \leqslant \mathrm{C}_{\mathrm{k}}$;

then equation (1.1) has

(i) a unique solution in $\mathrm{PC}\left(\mathcal{T}_{\mathcal{S}}{ }^{0} \cap\left[\mathrm{t}_{0}-\lambda, \mathrm{t}_{0}\right], \mathbb{R}^{\mathrm{m}}\right) \cap \mathrm{PC}^{1}\left(\mathcal{T}_{\mathcal{S}}{ }^{0}, \mathbb{R}^{\mathrm{m}}\right)$;

(ii) Hyers-Ulam stability on $\mathcal{T}_{\mathcal{S}}{ }^{0} \cap\left[\mathrm{t}_{0}-\lambda, \mathrm{t}_{0}\right]$.

Proof.

(i) Define an operator $\Lambda: \operatorname{PC}\left(\mathcal{T}_{\mathcal{S}}^{0} \cap\left[\mathrm{t}_{0}-\lambda, \mathrm{t}_{0}\right], \mathbb{R}^{\mathrm{m}}\right) \rightarrow \mathrm{PC}\left(\mathcal{T}_{\mathcal{S}}^{0} \cap\left[\mathrm{t}_{0}-\lambda, \mathrm{t}_{0}\right], \mathbb{R}^{\mathrm{m}}\right)$ by

$$
(\Lambda z)(t)=\left\{\begin{array}{l}
\alpha(t), \quad t \in\left[t_{0}-\lambda, t_{0}\right] \\
\alpha\left(t_{0}\right)+\Psi_{M}\left(t, t_{0}\right) z_{0}+\int_{t_{0}}^{t} \Psi_{M}(t, \Theta(s)) \int_{t_{0}}^{s} \mathcal{K}(s, u, z(u), z(h(u))) \Delta u \Delta s, \quad t \in\left(t_{0}, t_{1}\right], \\
\alpha\left(t_{0}\right)+\Upsilon_{1}\left(z\left(t_{1}^{-}\right)\right)+\Psi_{M}\left(t, t_{0}\right) z_{0} \\
\quad+\int_{t_{0}}^{t} \Psi_{M}(t, \Theta(s)) \int_{t_{0}}^{s} \mathcal{K}(s, u, z(u), z(h(u))) \Delta u \Delta s, \quad t \in\left(t_{1}, t_{2}\right], \\
\quad+\sum_{j=1}^{2} \Upsilon_{j}\left(z\left(t_{j}^{-}\right)\right)+\Psi_{M}\left(t, t_{0}\right) z_{0} \\
\quad+\int_{t_{0}}^{t} \Psi_{M}(t, \Theta(s)) \int_{t_{0}}^{s} \mathcal{K}(s, u, z(u), z(h(u))) \Delta u \Delta s, \quad t \in\left(t_{2}, t_{3}\right], \\
\alpha\left(t_{0}\right)+\sum_{j=1}^{m} \Upsilon_{j}\left(z\left(t_{j}^{-}\right)\right)+\Psi_{M}\left(t, t_{0}\right) z_{0} \\
\quad+\int_{t_{0}}^{t} \Psi_{M}(t, \Theta(s)) \int_{t_{0}}^{s} \mathcal{K}(s, u, z(u), z(h(u))) \Delta u \Delta s, \quad t \in\left(t_{m}, t_{m+1}\right] .
\end{array}\right.
$$

We see that for any $z_{1}, z_{2} \in \operatorname{PC}\left(\mathcal{T}_{\mathcal{S}}^{0} \cap\left[t_{0}-\lambda, t_{0}\right], \mathbb{R}^{\mathrm{m}}\right)$ and for all $t \in\left[\mathrm{t}_{0}-\lambda, \mathrm{t}_{0}\right]$, we have

$$
\left\|\left(\wedge z_{1}\right)(t)-\left(\wedge z_{2}\right)(t)\right\|=0 .
$$

For $t \in\left(t_{m}, t_{m+1}\right]$ consider,

$$
\begin{aligned}
& \left\|\left(\wedge z_{1}\right)(t)-\left(\Lambda z_{2}\right)(t)\right\|=\sum_{j=1}^{m}\left\|\Upsilon_{j}\left(z_{1}\left(t_{j}^{-}\right)\right)-\Upsilon_{j}\left(z_{2}\left(t_{j}^{-}\right)\right)\right\| \\
& +\| \int_{\mathrm{t}_{0}}^{\mathrm{t}} \Psi_{\mathrm{M}}(\mathrm{t}, \Theta(\mathrm{s})) \int_{\mathrm{t}_{0}}^{\mathrm{s}}\left(\mathcal{K}\left(\mathrm{s}, \mathrm{u}, z_{1}(\mathrm{u}), z_{1}(\mathrm{~h}(\mathrm{u}))\right)\right. \\
& \left.-\mathcal{K}\left(s, u, z_{2}(u), z_{2}(h(u))\right)\right) \Delta u \Delta s \| \\
& \leqslant \sum_{j=1}^{m} M_{j}\left\|z_{1}\left(t_{j}^{-}\right)-z_{2}\left(t_{j}^{-}\right)\right\|
\end{aligned}
$$




$$
\begin{aligned}
& +\sup _{\mathrm{t} \in \mathcal{T}_{\mathcal{S}}^{0} \cap\left[\mathrm{t}_{0}-\lambda, \mathrm{t}_{0}\right]} \int_{\mathrm{t}_{0}}^{\mathrm{t}}\left\|\Psi_{M}(\mathrm{t}, \Theta(s))\right\| \int_{\mathrm{t}_{0}}^{\mathrm{s}} \|\left(\mathcal{K}\left(\mathrm{s}, \mathrm{u}, z_{1}(\mathrm{u}), z_{1}(\mathrm{~h}(\mathrm{u}))\right)\right. \\
& \left.-\mathcal{K}\left(\mathrm{s}, \mathrm{u}, z_{2}(\mathrm{u}), z_{2}(\mathrm{~h}(\mathrm{u}))\right)\right) \| \Delta \mathrm{u} \Delta \mathrm{s} \\
& \leqslant \sum_{j=1}^{m} M_{j} \sup _{t \in \mathcal{T}_{\delta}^{0} \cap\left[\mathrm{t}_{0}-\lambda, \mathrm{t}_{0}\right]}\left\|z_{1}\left(\mathrm{t}_{\mathbf{j}}^{-}\right)-z_{2}\left(\mathrm{t}_{\mathbf{j}}^{-}\right)\right\| \\
& +\sup _{\mathrm{t} \in \mathcal{T}_{\mathcal{s}}^{0} \cap\left[\mathrm{t}_{0}-\lambda, \mathrm{t}_{0}\right]} \int_{\mathrm{t}_{0}}^{\mathrm{t}}\left\|\Psi_{M}(\mathrm{t}, \Theta(s))\right\| \int_{\mathrm{t}_{0}}^{\mathrm{s}} \mathrm{L}\left\|z_{1}(\mathrm{u})-z_{2}(\mathrm{u})\right\| \Delta \mathrm{u} \Delta \mathrm{s} \\
& +\sup _{\mathrm{t} \in \mathcal{T}_{\mathcal{S}} 0 \cap\left[\mathrm{t}_{0}-\lambda, \mathrm{t}_{0}\right]} \int_{\mathrm{t}_{0}}^{\mathrm{t}}\left\|\Psi_{M}(\mathrm{t}, \Theta(\mathrm{s}))\right\| \int_{\mathrm{t}_{0}}^{s} \mathrm{~L}\left\|z_{1}(\mathrm{~h}(\mathrm{u}))-z_{2}(\mathrm{~h}(\mathrm{u}))\right\| \Delta \mathrm{u} \Delta \mathrm{s} \\
& \leqslant \sum_{j=1}^{m} M_{j}\left\|z_{1}-z_{2}\right\|+2\left\|z_{1}-z_{2}\right\| \sup _{t \in \mathcal{T}_{s} 0 \cap\left[t_{0}-\lambda, t_{0}\right]} \int_{t_{0}}^{t}\left\|\Psi_{M}(t, \Theta(s))\right\| \int_{t_{0}}^{s} L \Delta u \Delta s \\
& \leqslant\left\|z_{1}-z_{2}\right\|\left(\sum_{j=1}^{m} M_{j}+2 \sup _{t \in \mathcal{T}_{\mathcal{S}}^{0} \cap\left[\mathrm{t}_{0}-\lambda, \mathrm{t}_{0}\right]} \int_{\mathrm{t}_{0}}^{\mathrm{t}}\left\|\Psi_{M}(\mathrm{t}, \Theta(s))\right\| \int_{\mathrm{t}_{0}}^{s} \mathrm{~L} \Delta \mathrm{u} \Delta s\right) .
\end{aligned}
$$

Following from (c), the operator is strictly contractive and hence a Picard operator on

$$
\operatorname{PC}\left(\mathcal{T}_{\mathcal{S}}^{0} \cap\left[t_{0}-\lambda, t_{0}\right], \mathbb{R}^{m}\right) .
$$

From (3.1), it follows that the unique fixed point of this operator is in fact the unique solution of (1.1) in $P \mathbb{C}\left(\mathcal{T}_{\mathcal{S}}{ }^{0} \cap\left[t_{0}-\lambda, t_{0}\right], \mathbb{R}^{m}\right) \cap P \mathbb{C}^{1}\left(\mathcal{T}_{\mathcal{S}}^{0}, \mathbb{R}^{m}\right)$.

(ii) Now let $y \in P C\left(\mathcal{T}_{\mathcal{S}}{ }^{0} \cap\left[t_{0}-\lambda, t_{0}\right], \mathbb{R}^{m}\right) \cap P C^{1}\left(\mathcal{T}_{\mathcal{S}}{ }^{0}, \mathbb{R}^{m}\right)$ be a solution to (2.1). The unique solution $z \in \mathrm{PC}\left(\mathcal{T}_{\mathcal{S}}^{0} \cap\left[\mathrm{t}_{0}-\lambda, \mathrm{t}_{0}\right], \mathbb{R}^{\mathrm{m}}\right) \cap \mathrm{PC}^{1}\left(\mathcal{T}_{\mathcal{S}}^{0}, \mathbb{R}^{\mathrm{m}}\right)$ of the dynamic equation

$$
\left\{\begin{array}{l}
z^{\Delta}(\mathrm{t})=M(\mathrm{t}) z(\mathrm{t})+\int_{\mathrm{t}_{0}}^{\mathrm{t}} \mathcal{K}(\mathrm{t}, \mathrm{s}, z(\mathrm{~s}), z(\mathrm{~h}(\mathrm{~s}))) \Delta s, \quad \mathrm{t} \in \mathcal{T}_{\mathcal{S}}{ }^{\prime}=\mathcal{T}_{\mathcal{S}}{ }^{0} \backslash\left\{\mathrm{t}_{1}, \mathrm{t}_{2} \ldots, \mathrm{t}_{\mathrm{m}}\right\}, \\
\Delta z\left(\mathrm{t}_{\mathrm{k}}\right)=z\left(\mathrm{t}_{\mathrm{k}}^{+}\right)-z\left(\mathrm{t}_{\mathrm{k}}^{-}\right)=\Upsilon_{\mathrm{k}}\left(z\left(\mathrm{t}_{\mathrm{k}}^{-}\right)\right), \quad \mathrm{k}=1,2, \cdots, \mathrm{m}, \\
z(\mathrm{t})=y(\mathrm{t}), \quad \mathrm{t} \in\left[\mathrm{t}_{0}-\lambda, \mathrm{t}_{0}\right], \\
z\left(\mathrm{t}_{0}\right)=y\left(\mathrm{t}_{0}\right)=z_{0},
\end{array}\right.
$$

is given by

$$
z(t)=\left\{\begin{array}{l}
y(t), \quad t \in\left[t_{0}-\lambda, t_{0}\right], \\
y\left(t_{0}\right)+\Psi_{M}\left(t, t_{0}\right) z_{0}+\int_{t_{0}}^{t} \Psi_{M}(t, \Theta(s)) \int_{t_{0}}^{s} \mathcal{K}(s, u, z(u), z(h(u))) \Delta u \Delta s, \quad t \in\left(t_{0}, t_{1}\right], \\
y\left(t_{0}\right)+\Upsilon_{1}\left(z\left(t_{1}^{-}\right)\right)+\Psi_{M}\left(t, t_{0}\right) z_{0}+\int_{t_{0}}^{t} \Psi_{M}(t, \Theta(s)) \int_{t_{0}}^{s} \mathcal{K}(s, u, z(u), z(h(u))) \Delta u \Delta s, \quad t \in\left(t_{1}, t_{2}\right], \\
y\left(t_{0}\right)+\sum_{j=1}^{2} \Upsilon_{j}\left(z\left(t_{j}^{-}\right)\right)+\Psi_{M}\left(t, t_{0}\right) z_{0}+\int_{t_{0}}^{t} \Psi_{M}(t, \Theta(s)) \int_{t_{0}}^{s} \mathcal{K}(s, u, z(u), z(h(u))) \Delta u \Delta s, \quad t \in\left(t_{2}, t_{3}\right], \\
\quad \vdots \\
y\left(t_{0}\right)+\sum_{j=1}^{m} \Upsilon_{j}\left(z\left(t_{j}^{-}\right)\right)+\Psi_{M}\left(t, t_{0}\right) z_{0} \\
\quad+\int_{t_{0}}^{t} \Psi_{M}(t, \Theta(s)) \int_{t_{0}}^{s} \mathcal{K}(s, u, z(u), z(h(u))) \Delta u \Delta s, \quad t \in\left(t_{m}, t_{m+1}\right] .
\end{array}\right.
$$


We observe that for all $t \in\left[t_{0}-\lambda, t_{0}\right]$, we have $\|y(t)-z(t)\|=0$. For $t \in\left(t_{m}, t_{m+1}\right]$, using Lemma 2.7 , we have

$$
\begin{aligned}
\|y(t)-z(t)\| \leqslant & \| y(t)-\Psi_{M}\left(t, t_{0}\right) y_{0}-\sum_{j=1}^{m} \Upsilon\left(y\left(t_{j}^{-}\right)\right) \\
& -\int_{t_{0}}^{t} \Psi_{M}(t, \Theta(s)) \int_{t_{0}}^{s} \mathcal{K}(s, u, y(u), y(h(u))) \Delta u \Delta s\left\|+\sum_{j=1}^{m}\right\| \Upsilon_{j}\left(y\left(t_{j}^{-}\right)\right)-\Upsilon_{j}\left(z\left(t_{j}^{-}\right)\right) \| \\
& +\left\|\int_{t_{0}}^{t} \Psi_{M}(t, \Theta(s)) \int_{t_{0}}^{s}(\mathcal{K}(s, u, y(u), y(h(u)))-\mathcal{K}(s, u, z(u), z(h(u)))) \Delta u \Delta s\right\| \\
\leqslant & \left(m+t_{f}-t_{0}\right) \epsilon+\sum_{j=1}^{m} M_{j}\left\|y\left(t_{j}^{-}\right)-z\left(t_{j}^{-}\right)\right\| \\
& +\int_{t_{0}}^{t}\left\|\Psi_{M}(t, \Theta(s))\right\| \int_{t_{0}}^{s} L\|y(u)-z(u)\| \Delta u \Delta s \\
& +\int_{t_{0}}^{t}\left\|\Psi_{M}(t, \Theta(s))\right\| \int_{t_{0}}^{s} L\|y(h(u))-z(h(u))\| \Delta u \Delta s .
\end{aligned}
$$

Next, we show that the operator $\mathrm{T}: \mathrm{PC}\left(\mathcal{T}_{\mathcal{S}}^{0} \cap\left[\mathrm{t}_{0}-\lambda, \mathrm{t}_{0}\right], \mathbb{R}^{\mathrm{m}}\right) \rightarrow \operatorname{PC}\left(\mathcal{T}_{\mathcal{S}}{ }^{0} \cap\left[\mathrm{t}_{0}-\lambda, \mathrm{t}_{0}\right], \mathbb{R}^{\mathrm{m}}\right)$ given below is an increasing Picard operator on $\operatorname{PC}\left(\mathcal{T}_{\mathcal{S}}^{0} \cap\left[\mathrm{t}_{0}-\lambda, \mathrm{t}_{0}\right], \mathbb{R}^{\mathrm{m}}\right)$.

$$
\begin{aligned}
& (\operatorname{Tg})(t)=\left\{\begin{array}{l}
0, \quad t \in\left[t_{0}-\lambda, t_{0}\right], \\
\left(t_{f}-t_{0}\right) \epsilon+\int_{t_{0}}^{t}\left\|\Psi_{M}(t, \Theta(s))\right\| \int_{t_{0}}^{s} \operatorname{Lg}(u) \Delta u \Delta s \\
\quad+\int_{t_{0}}^{t}\left\|\Psi_{M}(t, \Theta(s))\right\| \int_{t_{0}}^{s} \operatorname{Lg}(h(u)) \Delta u \Delta s, \quad t \in\left(t_{0}, t_{1}\right], \\
\left(1+t_{f}-t_{0}\right) \epsilon+M_{1} g\left(t_{1}^{-}\right)+\int_{t_{0}}^{t}\left\|\Psi_{M}(t, \Theta(s))\right\| \int_{t_{0}}^{s} \operatorname{Lg}(u) \Delta u \Delta s \\
\quad+\int_{t_{0}}^{t}\left\|\Psi_{M}(t, \Theta(s))\right\| \int_{t_{0}}^{s} \operatorname{Lg}(h(u)) \Delta u \Delta s, \quad t \in\left(t_{1}, t_{2}\right], \\
\left(2+t_{f}-t_{0}\right) \epsilon+\sum_{j=1}^{2} M_{j} g\left(t_{j}^{-}\right)+\int_{t_{0}}^{t}\left\|\Psi_{M}(t, \Theta(s))\right\| \int_{t_{0}}^{s} L g(u) \Delta u \Delta s
\end{array}\right. \\
& +\int_{t_{0}}^{t}\left\|\Psi_{M}(t, \Theta(s))\right\| \int_{t_{0}}^{s} \operatorname{Lg}(h(u)) \Delta u \Delta s, \quad t \in\left(t_{2}, t_{3}\right], \\
& \left(m+t_{f}-t_{0}\right) \epsilon+\sum_{j=1}^{m} M_{j} g\left(t_{j}^{-}\right)+\int_{t_{0}}^{t}\left\|\Psi_{M}(t, \Theta(s))\right\| \int_{t_{0}}^{s} L g(u) \Delta u \Delta s \\
& +\int_{t_{0}}^{t}\left\|\Psi_{M}(t, \Theta(s))\right\| \int_{t_{0}}^{s} \operatorname{Lg}(h(u)) \Delta u \Delta s, \quad t \in\left(t_{m}, t_{m+1}\right] .
\end{aligned}
$$

For any $g_{1}, g_{2} \in \operatorname{PC}\left(\mathcal{T}_{\mathcal{S}}^{0} \cap\left[t_{0}-\lambda, t_{0}\right], \mathbb{R}^{m}\right),\left\|\left(T g_{1}\right)(t)-\left(T g_{2}\right)(t)\right\|=0$ for all $t \in\left[t_{0}-\lambda, t_{0}\right]$. For $t \in$ $\left(t_{m}, t_{m+1}\right)$, consider

$$
\left\|\left(T g_{1}\right)(t)-\left(T g_{2}\right)(t)\right\| \leqslant \sum_{j=1}^{m} M_{j}\left\|g_{1}\left(t_{j}^{-}\right)-g_{2}\left(t_{j}^{-}\right)\right\|+\int_{t_{0}}^{t}\left\|\Psi_{M}(t, \Theta(s))\right\| \int_{t_{0}}^{s} L\left\|g_{1}(u)-g_{2}(u)\right\| \Delta u \Delta s
$$




$$
\begin{aligned}
& +\int_{t_{0}}^{t}\left\|\Psi_{M}(t, \Theta(s))\right\| \int_{t_{0}}^{s} L\left\|g_{1}(h(u))-g_{2}(h(u))\right\| \Delta u \Delta s \\
\leqslant & \sum_{j=1}^{m} M_{j} \sup _{t \in \mathcal{T}_{s}^{0} \cap\left[t_{0}-\lambda, t_{0}\right]}\left\|g_{1}\left(t_{j}^{-}\right)-g_{2}\left(t_{j}^{-}\right)\right\| \\
& +\int_{t_{0}}^{t}\left\|\Psi_{M}(t, \Theta(s))\right\| \int_{t_{0}}^{s} L \sup _{t \in \mathcal{T}_{s}^{0} \cap\left[t_{0}-\lambda, t_{0}\right]}\left\|g_{1}(u)-g_{2}(u)\right\| \Delta u \Delta s \\
& +\int_{t_{0}}^{t}\left\|\Psi_{M}(t, \Theta(s))\right\| \int_{t_{0}}^{s} L \sup _{t \in \mathcal{T}_{s}^{0} \cap\left[t_{0}-\lambda, t_{0}\right]}\left\|g_{1}(h(u))-g_{2}(h(u))\right\| \Delta u \Delta s \\
\leqslant & \sum_{j=1}^{m} M_{j}\left\|g_{1}-g_{2}\right\|+2\left\|g_{1}-g_{2}\right\| \sup _{t \in \mathcal{T}_{s}^{0} \cap\left[t_{0}-\lambda, t_{0}\right]} \int_{t_{0}}^{t}\left\|\Psi_{M}(t, \Theta(s))\right\| \int_{t_{0}}^{s} L \Delta u \Delta s \\
\leqslant & \left\|g_{1}-g_{2}\right\|\left(\sum_{j=1}^{m} M_{j}+2 \underset{t \in \mathcal{T}_{s}{ }^{0} \cap\left[t_{0}-\lambda, t_{0}\right]}{\sup _{t_{0}}}\left\|\Psi_{M}(t, \Theta(s))\right\| \int_{t_{0}}^{s} L \Delta u \Delta s\right) .
\end{aligned}
$$

Since $\left(\sum_{j=1}^{m} M_{j}+2 \sup _{t \in \mathcal{T}_{s}{ }^{0} \cap\left[t_{0}-\lambda, t_{0}\right]} \int_{t_{0}}^{t}\left\|\Psi_{M}(t, \Theta(s))\right\| \int_{t_{0}}^{s} L \Delta u \Delta s\right)<1$, so the operator is contractive on $\operatorname{PC}\left(\mathcal{T}_{\mathcal{S}}{ }^{0} \cap\left[t_{0}-\lambda, t_{0}\right], \mathbb{R}^{m}\right)$. Applying Banach contraction principle, $T$ is Picard operator with unique fixed point $g^{*} \in \operatorname{PC}\left(\mathcal{T}_{\mathcal{S}}^{0} \cap\left[t_{0}-\lambda, t_{0}\right], \mathbb{R}^{m}\right)$, i.e.,

$$
\begin{aligned}
g^{*}(t)= & \left(m+t_{f}-t_{0}\right) \epsilon+\sum_{j=1}^{m} M_{j} g^{*}\left(t_{j}^{-}\right)+\int_{t_{0}}^{t}\left\|\Psi_{M}(t, \Theta(s))\right\| \int_{t_{0}}^{s} L^{*}(u) \Delta u \Delta s \\
& +\int_{t_{0}}^{t}\left\|\Psi_{M}(t, \Theta(s))\right\| \int_{t_{0}}^{s} L g^{*}(h(u)) \Delta u \Delta s .
\end{aligned}
$$

Since $g^{*}$ is increasing, so $g^{*}(h(u)) \leqslant g^{*}(u)$ and by using $(d)$, we have

$$
g^{*}(t) \leqslant\left(m+t_{f}-t_{0}\right) \epsilon+\sum_{j=1}^{m} M_{j} g^{*}\left(t_{j}^{-}\right)+2 \int_{t_{0}}^{t} \int_{t_{0}}^{s} C_{k} L g^{*}(u) \Delta u \Delta s .
$$

By Lemma 2.4, we get

$$
g^{*}(t) \leqslant\left(m+t_{f}-t_{0}\right) \epsilon \prod_{t_{0}<t_{j}<t}\left(1+M_{j}\right) e_{P}\left(t, t_{0}\right)
$$

where $P(s)=2 \int_{t_{0}}^{s} C_{k} L \Delta u$. If we set $g(t)=\|y(t)-z(t)\|$, then from (3.2), $g(t) \leqslant(T g)(t)$ from which by using abstract Grönwall lemma, it follows that $g(t) \leqslant g^{*}(t)$, thus

$$
\|y(t)-z(t)\| \leqslant\left(m+t_{f}-t_{0}\right) \epsilon \prod_{t_{0}<t_{j}<t}\left(1+M_{j}\right) e_{p}\left(t, t_{0}\right) .
$$

Similarly, by following the same process, we can prove that:

\section{Theorem 3.2. If}

(a) The function $\mathcal{K}$ is piecewise continuous with the Lipschitz condition $\left\|\mathcal{K}\left(t, s, x_{1}, x_{2}\right)-\mathcal{K}\left(t, s, y_{1}, y_{2}\right)\right\| \leqslant$ $\sum_{i=1}^{2} L\left\|x_{i}-y_{i}\right\|, L>0$ for $t_{0} \leqslant s \leqslant t \leqslant t_{f}$ and for all $x_{i}, y_{i} \in \mathbb{R}^{m}, i \in\{1,2\} ;$

(b) $\Upsilon_{k}: \mathbb{R} \rightarrow \mathbb{R}$ is such that $\left\|\Upsilon_{k}\left(x_{1}\right)-\Upsilon_{k}\left(x_{2}\right)\right\| \leqslant M_{k}\left\|x_{1}-x_{2}\right\|, M_{k}>0$, for all $k \in\{1,2, \cdots, m\}$ and $x_{1}, x_{2} \in \mathbb{R}, i \in\{1,2\}$; 
(c) $\left(\sum_{j=1}^{m} M_{j}+2 \sup _{t \in \mathcal{T}_{\mathcal{S}}^{0} \cap\left[\mathrm{t}_{0}-\lambda, \mathrm{t}_{0}\right]} \int_{\mathrm{t}_{0}}^{\mathrm{t}}\left\|\Psi_{M}(\mathrm{t}, \Theta(s))\right\| \int_{\mathrm{t}_{0}}^{s} \mathrm{~L} \Delta \mathrm{u} \Delta \mathrm{s}\right)<1$;

(d) for some $\mathrm{C}_{\mathrm{k}} \geqslant 0$, we have $\left\|\Psi_{M}(\mathrm{t}, \Theta(\mathrm{s}))\right\|=\sup _{\mathrm{t} \in \mathcal{T}_{\mathrm{S}}^{0} \cap\left[\mathrm{t}_{0}-\lambda, \mathrm{t}_{0}\right]}\left\|\Psi_{M}(\mathrm{t}, \Theta(\mathrm{s}))\right\| \leqslant \mathrm{C}_{\mathrm{k}}$;

(e) $\varphi \in \mathbb{C}\left(\mathcal{T}_{\mathcal{S}}{ }^{0}, \mathbb{R}^{\mathrm{m}}\right)$ is increasing such that for some $\rho>0$,

$$
\int_{\mathrm{t}_{0}}^{\mathrm{t}} \varphi(\mathrm{r}) \Delta \mathrm{r} \leqslant \rho \varphi(\mathrm{t})
$$

then (1.1) has

(i) a unique solution in $\mathrm{PC}\left(\mathcal{T}_{\mathcal{S}}^{0} \cap\left[\mathrm{t}_{0}-\lambda, \mathrm{t}_{0}\right], \mathbb{R}^{\mathrm{m}}\right) \cap \mathrm{PC}^{1}\left(\mathcal{T}_{\mathcal{S}}{ }^{0}, \mathbb{R}^{\mathrm{m}}\right)$;

(ii) Hyers-Ulam-Rassias stability on $\mathcal{T}_{\mathcal{S}}^{0} \cap\left[\mathrm{t}_{0}-\lambda, \mathrm{t}_{0}\right]$.

\section{Conclusion}

In this paper, we have proved the Hyers-Ulam stability and Hyers-Ulam-Rassias stability of (1.1) using fixed point method. We proved our results by using abstract Grönwall lemma together with Lemma 2.4. Moreover, our results guarantee that there is an exact solution of (1.1) which is close to the approximate solution.

\section{Acknowledgment}

This work was supported by the National Natural Science Foundation of China (11571378).

\section{References}

[1] R. P. Agarwal, A. S. Awan, D. O'Regan, A. Younus, Linear impulsive Volterra integro-dynamic system on time scales, Adv. Difference Equ., 2014 (2014), 17 pages. 1

[2] C. Alsina, R. Ger, On some inequalities and stability results related to the exponential function, J. Inequal. Appl., 2 (1998), 373-380. 1

[3] S. András, A. R. Mészáros, Ulam-Hyers stability of dynamic equations on time scales via Picard operators, Appl. Math. Comput., 209 (2013), 4853-4864. 1

[4] D. D. Baĭnov, A. B. Dishliev, Population dynamics control in regard to minimizing the time necessary for the regeneration of a biomass taken away from the population, C. R. Acad. Bulgare Sci., 42 (1989), 29-32. 1

[5] M. Bohner, A. Peterson, Dynamic equations on time scales: An introduction with applications, Birkhäuser Boston, USA, (2001). 1

[6] M. Bohner, A. Peterson, Advances in Dynamics Equations on time scales, Birkhäuser Boston, USA, (2003).

[7] J. J. Dacunha, Stability for time varying linear dynamic systems on time scales, J. Comput. Appl. Math., 176 (2005), 381-410.

[8] A. E. Hamza, K. M. Oraby, Stability of abstract dynamic equations on time scales, Adv. Difference Equ., 2012 (2012), 15 pages. 1

[9] S. Hilger, Analysis on measure chains-A unified approach to continuous and discrete calculus, Result math., 18 (1990), 18-56. 1

[10] D. H. Hyers, On the stability of the linear functional equation, Proc. Nat. Acad. Sci. U.S.A., 27 (1941), 222-224. 1

[11] S.-M. Jung, Hyers-Ulam stability of linear differential equations of first order, Appl. Math. Lett., 17 (2004), 1135-1140. 1

[12] S.-M. Jung, Hyers-Ulam stability of a system of first order linear differential equations with constant coefficients, J. Math. Anal. Appl., 320 (2006), 549-561. 1

[13] S.-M. Jung, Hyers-Ulam stability of linear partial differential equations of first order, Appl. Math. Lett., 22 (2009), 70-74.

[14] S.-M. Jung, J. Roh, The linear differential equations with complex constant coefficients and Schrödinger equations, Appl. Math. Lett., 66 (2017), 23-29. 1

[15] Y.-J. Li, Y. Shen, Hyers-Ulam stability of linear differential equations of second order, Appl. Math. Lett., 23 (2010), 306-309. 1

[16] T.-X. Li, A. Zada, Connections between Hyers-Ulam stability and uniform exponential stability of discrete evolution families of bounded linear operators over Banach spaces, Adv. Difference Equ., 2016 (2016), 8 pages. 1 
[17] V. Lupulescu, A. Zada, Linear impulsive dynamic systems on time scales, Electron. J. Qual. Theory Differ. Equ., 2010 (2010), 30 pages. $1,2.4$

[18] M. Obłoza, Hyers stability of the linear differential equation, Rocznik Nauk.-Dydakt. Prace Mat., 13 (1993), 259-270. 1

[19] M. Obłoza, Connections between Hyers and Lyapunov stability of the ordinary differential equations, Rocznik Nauk.Dydakt. Prace Mat., 14 (1997), 141-146. 1

[20] C. Pötzsche, S. Siegmund, F. Wirth, A spectral characterization of exponential stability for linear time-invariant systems on time scales, Discrete Contin. Dyn. Sys., 9 (2003), 1223-1241. 1

[21] T. M. Rassias, On the stability of linear mappings in Banach spaces, Proc. Amer. Math. Soc., 72 (1978), 297-300. 1

[22] I. A. Rus, Gronwall lemmas: ten open problems, Sci. Math. Jpn., 70 (2009), 221-228. 2.5

[23] S. M. Ulam, A collection of the mathematical problems, Interscience Publishers, New York, (1960). 1

[24] S. M. Ulam, Problem in modern mathematics, Science Editions John Wiley and Sons, New York, (1964). 1

[25] A. Zada, W. Ali, S. Farina, Hyers-Ulam stability of nonlinear differential equations with fractional integrable impulses, Math. Methods Appl. Sci., 40 (2017), 5502-5514. 1

[26] A. Zada, S. Faisal, Y.-J. Li, On the Hyers-Ulam stability of first-order impulsive delay differential equations, J. Funct. Spaces, 2016 (2016), 6 pages. 1

[27] A. Zada, T.-X. Li, S. Ismail, O. Shah, Exponential dichotomy of linear autonomous systems over time scales, Diff. Equa. Appl., 8 (2016), 123-134. 1

[28] A. Zada, S. O. Shah, S. Ismail, T.-X. Li, Hyers-Ulam stability in terms of dichotomy of first order linear dynamic systems, Punjab Univ. J. Math., 49 (2017), 37-47. 1

[29] A. Zada, O. Shah, R. Shah, Hyers-Ulam stability of non-autonomous systems in terms of boundedness of Cauchy problems, Appl. Math. Comput., 271 (2015), 512-518.

[30] A. Zada, F. Ullah Khan, U. Riaz, T.-X. Li, Hyers-Ulam stability of linear summation equations, Punjab Univ. J. Math., 49 (2017), 19-24.

[31] A. Zada, P. Wang, D. Lassoued, T.-X. Li, Connections between Hyers-Ulam stability and uniform exponential stability of 2-periodic linear nonautonomous systems, Adv. Difference Equ., 2017 (2017), 7 pages. 1 